MATHEMATICS OF COMPUTATION

Volume 72, Number 244, Pages 1947-1961

S 0025-5718(03)01560-6

Article electronically published on May 14, 2003

\title{
INDEX-DOUBLING IN SEQUENCES BY AITKEN EXTRAPOLATION
}

\author{
ROGER ALEXANDER
}

\begin{abstract}
Aitken extrapolation, applied to certain sequences, yields the evennumbered subsequence of the original. We prove that this is true for sequences generated by iterating a linear fractional transformation, and for some sequences of convergents of the regular continued fractions of certain quadratic irrational numbers.
\end{abstract}

\section{Introduction}

Consider the sequence generated by iteration of a linear fractional transformation,

$$
C_{1}=A_{1} / B_{1}, \quad C_{n+1}=\frac{A_{1} C_{n}+A_{1}^{\prime}}{B_{1} C_{n}+B_{1}^{\prime}}, \quad n \geq 1 .
$$

Assume $B_{1} \neq 0$ and $\delta \equiv A_{1} B_{1}^{\prime}-A_{1}^{\prime} B_{1} \neq 0$. We prove in $\S 2$ below that applying Aitken extrapolation with any step length $k$ to this sequence, that is, computing

$$
C_{n}^{k *}=\frac{C_{n+k} C_{n-k}-C_{n}^{2}}{C_{n+k}-2 C_{n}+C_{n-k}}, \quad k<n,
$$

produces the even-indexed subsequence:

$$
C_{n}^{k *}=C_{2 n}
$$

This result implies that applying Aitken extrapolation again to the resulting evenindexed subsequence produces the subsequence with indices divisible by four, and so on for all powers of two.

In $\S 3$ we consider sequences of convergents $C_{n}$ of certain regular continued fractions that represent quadratic irrational numbers:

$$
C_{n}=b_{1}+\frac{1}{b_{2}+\frac{1}{b_{3}+\cdot \frac{1}{b_{n-1}+\frac{1}{b_{n}}}}} .
$$

Received by the editor January 4, 2002.

2000 Mathematics Subject Classification. Primary 65B05, 11A55.

Key words and phrases. Aitken extrapolation, linear fractional transformation, periodic continued fraction.

(C)2003 American Mathematical Society 
In special cases these sequences can be generated by iterating a linear fractional transformation, but not in general. We derive necessary and sufficient conditions for the Aitken extrapolant

$$
C_{n}^{*}=\frac{C_{n+1} C_{n-1}-C_{n}^{2}}{C_{n+1}-2 C_{n}+C_{n-1}}
$$

to belong to the original sequence. For example, we prove that $C_{n}^{*}=C_{2 n}$ if the partial quotients $b_{k}$ form a purely periodic sequence with minimal period $\ell$, say, and the index $n$ is a multiple of $\ell$, or of $\ell / 2$ if $\ell$ is even. We also consider extrapolation with step lengths greater than one, and extrapolation in subsequences of $C_{n}$.

The theorems on extrapolation of convergents of continued fractions appear to be new. Several theorems on iterates of linear fractional transformations are known. Phillips [Ph 1984] proves that $\varphi_{k}^{*}=\varphi_{2 k}$ for the sequence defined by

$$
\varphi_{1}=1, \quad \varphi_{k+1}=1+1 / \varphi_{k}
$$

that converges to the golden ratio $\varphi$. Elements of the sequence are ratios of consecutive Fibonacci numbers; they are also the convergents of the regular continued fraction for $\varphi$.

McCabe and Phillips [MP 1985] prove the following, although they state it in a form different from that given here.

Theorem 1. Let the sequence $\left(C_{n}\right)_{n=1}^{\infty}$ be defined by

$$
C_{1}=b, \quad C_{n+1}=b-\frac{a}{C_{n}},
$$

and assume that $b \neq 0, a \neq 0$, and that $\cos ^{-1}\left(\frac{1}{2} b / \sqrt{a}\right)$ is not a rational multiple of $\pi$. Then Aitken extrapolation with any step length $p<n$,

$$
C_{n}^{p *}=\frac{C_{n+p} C_{n-p}-C_{n}^{2}}{C_{n+p}-2 C_{n}+C_{n-p}}
$$

yields $C_{n}^{p *}=C_{2 n}$.

This theorem implies that repeated Aitken extrapolation always "doubles the index," that is, extrapolating the even-indexed subsequence produces the subsequence with indices divisible by four, and so on. Brezinski and Lembarki BL 1986] proved the same for sequences defined by

$$
\begin{gathered}
C_{1}=b, \quad C_{n+1}=1+\frac{a}{C_{n}} . \\
\text { 2. EXTRAPOLATION OF SEQUENCES } \\
\text { GENERATED BY LINEAR FRACTIONAL TRANSFORMATIONS }
\end{gathered}
$$

The purpose of this section is to prove our first main theorem.

Theorem 2. Let $\left(C_{n}\right)_{n=1}^{\infty}$ be a sequence generated by a linear fractional transformation (1) such that $\delta \equiv A_{1} B_{1}^{\prime}-A_{1}^{\prime} B_{1} \neq 0$ and $B_{1} \neq 0$. Then for any $n>1$ the Aitken extrapolant with any step length $p<n$ is given by

$$
C_{n}^{p *}=C_{n}+\frac{C_{n+p}-C_{n}}{1-r_{n}^{(p)}}, \quad r_{n}^{(p)}=\frac{C_{n+p}-C_{n}}{C_{n}-C_{n-p}},
$$

and satisfies $C_{n}^{p *}=C_{2 n}$.

Repeated application of Theorem [2] with $p=2^{k}, k=0,1,2, \ldots$, allows us to conclude that repeated Aitken extrapolation "doubles the index every time." 
Corollary 1. For every $k \geq 0$, Aitken extrapolation of the subsequence $\left(C_{2^{k} n}\right)_{n=1}^{\infty}$ of the sequence (II) produces the subsequence $\left(C_{2^{k+1} n}\right)_{n=1}^{\infty}$.

Before giving the proof we derive several preliminary results, beginning with the fact that Aitken extrapolation is correctly expressed by (3).

Given a sequence $x_{1}, x_{2}, x_{3}, \ldots$ supposed (truly or not) to be linearly convergent, one may compute estimates $r_{n}$ of the convergence rate by

$$
r_{n}:=\frac{x_{n+1}-x_{n}}{x_{n}-x_{n-1}}
$$

and extrapolate by

$$
x_{n}^{*}=x_{n}+\frac{1}{1-r_{n}}\left(x_{n+1}-x_{n}\right) .
$$

It is easily verified that this expression is equivalent to the standard one, $x_{n}^{*}=$ $\left(x_{n+1} x_{n-1}-x_{n}^{2}\right) /\left(x_{n+1}-2 x_{n}+x_{n-1}\right)$, for Aitken extrapolation. Formulated this way, the algorithm can be understood as approximating the series

$$
\lim _{m \rightarrow \infty} x_{m}=x_{n}+\sum_{k=0}^{\infty}\left(x_{n+k+1}-x_{n+k}\right)
$$

by a geometric one, replacing each term $\left(x_{n+k+1}-x_{n+k}\right)$ by $r_{n}^{k}\left(x_{n+1}-x_{n}\right)$.

For future use let us record some facts about Aitken extrapolation that are immediately obvious from this formulation.

Lemma 1 ([Br 1991, $\S \S 1.1,1.4])$. Aitken extrapolation has the following properties.

(1) Aitken extrapolation of the sequence generated by the linear iteration $C_{n+1}$ $=A_{1} C_{n}+A_{1}^{\prime}$ with $C_{1}$ arbitrary produces "convergence" in a single step: $C_{n}^{*}=C_{\infty} \equiv A_{1}^{\prime} /\left(1-A_{1}\right)$. (This occurs whether the original sequence converges $\left(\left|A_{1}\right|<1\right)$ or diverges $\left(\left|A_{1}\right| \geq 1\right)$.)

(2) Aitken extrapolation commutes with translation of the sequence by a constant: if the sequence $D_{n}$ is defined by $D_{n}=C_{n}+a$, then the extrapolated sequences are related by $D_{n}^{*}=C_{n}^{*}+a$.

(3) Aitken extrapolation is homogeneous of degree one: if the sequence $D_{n}$ is defined by $D_{n}=a C_{n}$, then the extrapolated sequences are related by $D_{n}^{*}=$ $a C_{n}^{*}$.

The first property means that if $B_{1}=0$ in the linear fractional transformation (11), so that the iteration function is linear, then Aitken extrapolation produces the "limit" in a single step. We require $B_{1} \neq 0$ to avoid this uninteresting case.

Proof of Theorem 2 using Theorem 11. Given a sequence (1), we define a new sequence by

$$
D_{n}=B_{1} C_{n}+B_{1}^{\prime} .
$$

Then $D_{1}=B_{1}\left(A_{1} / B_{1}\right)+B_{1}^{\prime}=A_{1}+B_{1}^{\prime}$, and a calculation shows that

$$
D_{n+1}=\left(A_{1}+B_{1}^{\prime}\right)-\frac{A_{1} B_{1}^{\prime}-A_{1}^{\prime} B_{1}}{D_{n}} .
$$

Thus the sequence $D_{n}$ satisfies the hypotheses of Theorem[1, and we conclude that Aitken extrapolation gives

$$
D_{n}^{p *}=D_{2 n} .
$$


By Lemma 1 Aitken extrapolation commutes with translation and dilation. Therefore

$$
C_{n}^{p *}=\frac{D_{n}^{p *}-B_{1}^{\prime}}{B_{1}}=\frac{D_{2 n}-B_{1}^{\prime}}{B_{1}}=C_{2 n},
$$

and this completes the proof.

We present next a direct proof of Theorem 2 that is no longer than that just given if the proof of Theorem 1 be factored in, and that features techniques useful in the extension of our results to sequences of convergents of continued fractions. It will be advantageous to regard linear fractional transformations as mappings of the real projective line, in the familiar way. Let $M$ be the matrix with coefficients from (11),

$$
M=\left[\begin{array}{ll}
A_{1} & B_{1} \\
A_{1}^{\prime} & B_{1}^{\prime}
\end{array}\right]
$$

Lemma 2. Denote the elements of $M^{n}$ by

$$
M^{n}=\left[\begin{array}{cc}
A_{n} & B_{n} \\
A_{n}^{\prime} & B_{n}^{\prime}
\end{array}\right] .
$$

Then the iterates (1) satisfy

$$
C_{n}=A_{n} / B_{n}, \quad n \geq 1 .
$$

In (7) we understand $C_{n}=\infty$ when $B_{n}=0$; under our assumptions $A_{n}$ and $B_{n}$ do not vanish simultaneously.

Proof. The case $n=1$ is true by definition, and, assuming (17) is true for $n$, we have (writing $e_{i}, i=1,2$, for the standard unit vectors of $\mathbb{R}^{2}$ ) $\left[A_{n+1} B_{n+1}\right]=e_{1}^{T} M^{n} M=$ $\left[A_{n} B_{n}\right] M$, so that

$$
\frac{A_{n+1}}{B_{n+1}}=\frac{A_{1} A_{n}+A_{1}^{\prime} B_{n}}{B_{1} A_{n}+B_{1}^{\prime} B_{n}}=\frac{A_{1} C_{n}+A_{1}^{\prime}}{B_{1} C_{n}+B_{1}^{\prime}}=C_{n+1} .
$$

We may thus regard $\left(A_{n}, B_{n}\right)$ as homogeneous coordinates for $C_{n}$, and understand the iteration of the linear fractional transformation in terms of the related matrix power iteration.

Given a linear fractional transformation (1) with corresponding matrix $M$ (6), we shall find useful a certain factorization of $M$.

Lemma 3. Given a matrix $M$ (6) such that $B_{1} \neq 0$ and $\delta=\operatorname{det} M \neq 0$, let

$$
b=\frac{A_{1}-B_{1}^{\prime}}{B_{1}}, \quad V=\left[\begin{array}{cc}
b & 1 \\
1 & 0
\end{array}\right],
$$

Then $H=M V^{-1}$ is symmetric with $\operatorname{det} H=-\delta$. Furthermore, whenever a nonsingular matrix $M$ has a factorization $M=H V$ with $V$ as in (8) and $H$ symmetric, then for every $n \geq 1$,

$$
\left(M^{n} e_{2}\right)^{T} V M^{n}=e_{1}^{T} M^{2 n} .
$$

Proof. A calculation gives

$$
H=M V^{-1}=\left[\begin{array}{rr}
B_{1} & B_{1}^{\prime} \\
B_{1}^{\prime} & \frac{\left(B_{1}^{\prime}\right)^{2}-\delta}{B_{1}}
\end{array}\right],
$$


showing that $H$ is symmetric, $M=H V$, and $\operatorname{det} H=-\delta$. Additionally, since both $V$ and $H$ are symmetric,

$$
\begin{aligned}
\left(M^{n} e_{2}\right)^{T} V M^{n} & =e_{2}^{T}(V H)^{n} V(H V)^{n} \\
& =e_{2}^{T} V(H V)^{2 n}=e_{1}^{T} M^{2 n}
\end{aligned}
$$

Direct proof of Theorem Q Let $n>p \geq 1$ and calculate

$$
C_{n+p}-C_{n}=\frac{A_{n+p}}{B_{n+p}}-\frac{A_{n}}{B_{n}}=\frac{A_{n+p} B_{n}-A_{n} B_{n+p}}{B_{n+p} B_{n}}=-\frac{\delta^{n} B_{p}}{B_{n+p} B_{n}},
$$

using in the last numerator the determinant of the matrix

$$
\left[\begin{array}{rr}
A_{n+p} & B_{n+p} \\
A_{n} & B_{n}
\end{array}\right]=\left[\begin{array}{c}
e_{1}^{T} M^{n+p} \\
e_{1}^{T} M^{n}
\end{array}\right]=\left[\begin{array}{rr}
A_{p} & B_{p} \\
1 & 0
\end{array}\right] M^{n} .
$$

Replacing $n$ by $n-p$ in (11) gives

$$
C_{n}-C_{n-p}=-\frac{\delta^{n-p} B_{p}}{B_{n} B_{n-p}}
$$

so that the rate estimate (3) is $r_{n}^{(p)}=\delta^{p} B_{n-p} / B_{n+p}$, and extrapolation yields

$$
\begin{aligned}
C_{n}^{p *} & =\frac{A_{n}}{B_{n}}+\frac{1}{1-\delta^{p} B_{n-p} / B_{n+p}} \frac{A_{n+p} B_{n}-A_{n} B_{n+p}}{B_{n+p} B_{n}} \\
& =\frac{A_{n+p} B_{n}-\delta^{p} A_{n} B_{n-p}}{B_{n+p} B_{n}-\delta^{p} B_{n} B_{n-p}} .
\end{aligned}
$$

To simplify this last expression we use $b$ from (8) to define, for $n \geq 0$,

$$
A_{n}^{\prime \prime}=b A_{n}+A_{n}^{\prime}, \quad B_{n}^{\prime \prime}=b B_{n}+B_{n}^{\prime},
$$

so that

$$
\left[\begin{array}{ll}
A_{n}^{\prime \prime} & B_{n}^{\prime \prime} \\
A_{n} & B_{n}
\end{array}\right]=\left[\begin{array}{ll}
b & 1 \\
1 & 0
\end{array}\right]\left[\begin{array}{cc}
A_{n} & B_{n} \\
A_{n}^{\prime} & B_{n}^{\prime}
\end{array}\right]=V M^{n}
$$

If $X$ is any matrix, it will be convenient to write either $e_{i}^{T} X e_{j}$ or $X_{i j}$ for the $(i, j)$ element of $X$. In particular, when $k$ is any integer, we write $X_{i j}^{k}$ for the $(i, j)$ element of $X^{k}$. Then, using the factorization $M=H V$ and (15), we have

$$
\left[\begin{array}{cc}
A_{n+p} & B_{n+p} \\
A_{n+p}^{\prime} & B_{n+p}^{\prime}
\end{array}\right]=M^{p} M^{n}=M^{p-1} H V M^{n}=M^{p-1} H\left[\begin{array}{cc}
A_{n}^{\prime \prime} & B_{n}^{\prime \prime} \\
A_{n} & B_{n}
\end{array}\right],
$$

and thus

$$
\begin{aligned}
& A_{n+p}=\left(M^{p-1} H\right)_{11} A_{n}^{\prime \prime}+\left(M^{p-1} H\right)_{12} A_{n} \\
& B_{n+p}=\left(M^{p-1} H\right)_{11} B_{n}^{\prime \prime}+\left(M^{p-1} H\right)_{12} B_{n} .
\end{aligned}
$$

Shifting the index down by $p$ gives

$$
\left[\begin{array}{l}
B_{n} \\
B_{n}^{\prime}
\end{array}\right]=M^{p-1} H\left[\begin{array}{c}
B_{n-p}^{\prime \prime} \\
B_{n-p}
\end{array}\right] \quad \text { or } \quad\left[\begin{array}{l}
B_{n-p}^{\prime \prime} \\
B_{n-p}
\end{array}\right]=\left(M^{p-1} H\right)^{-1}\left[\begin{array}{c}
B_{n} \\
B_{n}^{\prime}
\end{array}\right],
$$

so that

$$
B_{n-p}=\left(M^{p-1} H\right)_{21}^{-1} B_{n}+\left(M^{p-1} H\right)_{22}^{-1} B_{n}^{\prime} .
$$


Now, since $M^{p-1} H$ is $2 \times 2$ with $\operatorname{det}\left(M^{p-1} H\right)=-\delta^{p}$, we have

$$
\left(M^{p-1} H\right)_{22}^{-1}=\frac{-1}{\delta^{p}}\left(M^{p-1} H\right)_{11}, \quad\left(M^{p-1} H\right)_{21}^{-1}=\frac{1}{\delta^{p}}\left(M^{p-1} H\right)_{21} .
$$

Inserting (16), (17) and (18) into formula (14) for $C_{n}^{p *}$ and using (19) gives

$$
C_{n}^{p *}=\frac{\left(M^{p-1} H\right)_{11}\left(A_{n}^{\prime \prime} B_{n}+A_{n} B_{n}^{\prime}\right)+\left(\left(M^{p-1} H\right)_{12}-\left(M^{p-1} H\right)_{21}\right) A_{n} B_{n}}{\left(M^{p-1} H\right)_{11}\left(B_{n}^{\prime \prime} B_{n}+B_{n} B_{n}^{\prime}\right)+\left(\left(M^{p-1} H\right)_{12}-\left(M^{p-1} H\right)_{21}\right) B_{n}^{2}}
$$

But $M^{p-1} H=(H V)^{p-1} H$ is symmetric, so the second terms in numerator and denominator vanish, leaving

$$
C_{n}^{p *}=\frac{A_{n}^{\prime \prime} B_{n}+A_{n} B_{n}^{\prime}}{B_{n}^{\prime \prime} B_{n}+B_{n} B_{n}^{\prime}} .
$$

We may write this as a quotient of inner products and apply (15),

$$
C_{n}^{p *}=\frac{\left[\begin{array}{cc}
B_{n} & B_{n}^{\prime}
\end{array}\right] \cdot\left[\begin{array}{c}
A_{n}^{\prime \prime} \\
A_{n}
\end{array}\right]}{\left[\begin{array}{ll}
B_{n} & B_{n}^{\prime}
\end{array}\right] \cdot\left[\begin{array}{c}
B_{n}^{\prime \prime} \\
B_{n}
\end{array}\right]}=\frac{\left(M^{n} e_{2}\right)^{T} V M^{n} e_{1}}{\left(M^{n} e_{2}\right)^{T} V M^{n} e_{2}} .
$$

Therefore $C_{n}^{p *}$ is expressed in homogeneous coordinates by the vector

$$
e_{2}^{T} M^{n}\left(V M^{n}\right),
$$

and by (9) of Lemma 3 this is $e_{1}^{T} M^{2 n}=\left[A_{2 n}, B_{2 n}\right]$.

\section{Extrapolation of CONVERGENTS OF REgUlaR CONTINUED FRACTIONS}

3.1. Regular continued fractions and quadratic irrationals. Recall [Pe 1929] $\S \S 1-25]$ that a regular continued fraction is an expression of the form

$$
b_{1}+\frac{1}{b_{2}+\frac{1}{b_{3}+\cdots}}=b_{1}+\frac{1 \mid}{\mid b_{2}}+\frac{1 \mid}{\mid b_{3}}+\cdots
$$

in which the $b_{i}$ are positive integers called the partial quotients. The convergents of the continued fraction are the terminating continued fractions

$$
C_{n}=b_{1}+\frac{1 \mid}{\mid b_{2}}+\frac{1 \mid}{\mid b_{3}}+\cdots+\frac{1 \mid}{\mid b_{n}}=\frac{A_{n}}{B_{n}}
$$

with integral numerators $A_{n}$ and denominators $B_{n}$ computable recursively by $A_{0}=$ $1, A_{-1}=0, B_{0}=0, B_{-1}=1$, and then, for $n=1,2, \ldots$,

$$
\begin{aligned}
& A_{n}=b_{n} A_{n-1}+A_{n-2}, \\
& B_{n}=b_{n} B_{n-1}+B_{n-2} .
\end{aligned}
$$

The continued fraction represents the irrational number $\gamma=\lim _{n \rightarrow \infty} C_{n}$.

Conversely, a positive irrational number $\gamma$ determines its sequence of partial quotients $b_{i}$ by $\gamma_{1}=\gamma$ and then (writing $\lfloor\cdot\rfloor$ for the greatest integer function)

$$
\left.\begin{array}{ccc}
b_{i} & = & \left\lfloor\gamma_{i}\right\rfloor, \\
\gamma_{i+1} & = & 1 /\left(\gamma_{i}-b_{i}\right)
\end{array}\right\}, \quad i=1,2, \ldots
$$

The sequence (21) mentioned in the Introduction is the sequence of convergents of the regular continued fraction for the golden ratio $\varphi=(1+\sqrt{5}) / 2$, a quadratic irrational number. Phillips' theorem $\mathrm{Ph} 1984$ asserts that Aitken extrapolation applied to this sequence produces the even-numbered subsequence. Such a result 
is not true for the sequence of convergents of arbitrary regular continued fractions. Consider the first eight convergents of the regular continued fraction for $\sqrt{7}$ :

$$
\left(C_{k}\right)_{k=1}^{8}=\left(2,3, \frac{5}{2}, \frac{8}{3}, \frac{37}{14}, \frac{45}{17}, \frac{82}{31}, \frac{127}{48}\right) .
$$

Then $C_{2}^{*}=\left(\frac{5}{2} \cdot 2-3^{2}\right) /\left(\frac{5}{2}-2 \cdot 3+2\right)=C_{4}$. However, $C_{3}^{*}=21 / 8$ is not only different from $C_{6}$ but does not appear at all in the sequence of convergents, while again $C_{4}^{*}=C_{8}$. In fact in this case $C_{2 k}^{*}=C_{4 k}$ but $C_{2 k+1}^{*} \neq C_{4 k+2}$ for $k=1,2, \ldots$, and the Aitken extrapolants of odd-numbered convergents are not themselves convergents.

Thus Aitken acceleration applied to the convergents of a regular continued fraction can produce convergents, or not. In this section we determine necessary and sufficient conditions for the result of Aitken extrapolation to belong to the sequence of convergents. We begin by recalling the properties of periodic regular continued fractions, which will play the main role in what follows.

A continued fraction is periodic (also called mixed periodic) if there exist a starting index $n_{0}$ and an integer $\ell \geq 1$ such that $b_{n+\ell}=b_{n}$ for all $n \geq n_{0}$. The shortest repeating sequence $\left(b_{n}, \ldots, b_{n+\ell-1}\right)$ is a primitive period. If the primitive period starts at $n_{0}=1$ the continued fraction is called purely periodic.

A number $\gamma$ has a periodic continued fraction, if and only if $\gamma$ is a quadratic irrational, that is, if and only if $\gamma$ can be written $\gamma=(P+\sqrt{D}) / Q$ with $P, Q, D \in \mathbb{Z}$, $D>0$ not the square of an integer, $Q \neq 0$ and $P^{2} \equiv D(\bmod Q)$. A quadratic irrational $\gamma>0$ is represented by a purely periodic continued fraction if and only if it is reduced: $\gamma>1$, and its conjugate $\gamma^{\prime}=(P-\sqrt{D}) / Q$ satisfies $-1<\gamma^{\prime}<0$.

We write $\left[b_{1}, \ldots, b_{k}\right]$ for the finite continued fraction with partial quotients $b_{i}$, and $\left[\overline{b_{1}, \ldots, b_{\ell}}\right]$ for the purely periodic infinite continued fraction with primitive period of length $\ell$.

We state first some well known properties of convergents of regular continued fractions [Pe 1929], in the form in which we use them here.

Lemma 4. Let $\left(b_{n}\right)_{n=1}^{\infty}$ be the sequence of partial quotients of a regular continued fraction. Define matrices $V_{n}$ for $n \geq 1$ and $M_{n}$ for $n \geq 0$ by

$$
V_{n}=\left[\begin{array}{cc}
b_{n} & 1 \\
1 & 0
\end{array}\right], \quad M_{n}=\left[\begin{array}{cc}
A_{n} & B_{n} \\
A_{n-1} & B_{n-1}
\end{array}\right],
$$

with $A_{n}$ and $B_{n}$ defined by the recursions (21) and (22). Then $M_{n}=V_{n} M_{n-1}=$ $V_{n} V_{n-1} \cdots V_{1}$ for $n \geq 1$, and the convergents satisfy

$$
C_{n}-C_{n-1}=\frac{(-1)^{n}}{B_{n} B_{n-1}} .
$$

Proof. The first statement is immediate from the recursions (21) and (22) defining the convergents. For the difference (24) we have

$$
C_{n}-C_{n-1}=A_{n} / B_{n}-A_{n-1} / B_{n-1}=\frac{A_{n} B_{n-1}-A_{n-1} B_{n}}{B_{n} B_{n-1}}=\frac{\operatorname{det} M_{n}}{B_{n} B_{n-1}},
$$

and, since $\operatorname{det} V_{n}=-1$ for all $n$, we have $\operatorname{det} M_{n}=(-1)^{n}$.

3.2. Extrapolation of period-ending convergents. We can now state the first result on Aitken extrapolation of convergents. When the length $\ell$ of the primitive period is greater than one, it is not true that the convergents are derived by iterating a linear fractional transformation. It is true, however, if we restrict our attention to the subsequence of period-ending convergents $\left(C_{n \ell}\right)_{n=1}^{\infty}$. 
Theorem 3. Let $\gamma$ be a reduced quadratic irrational number, let $\ell$ be the length of the primitive period of its regular continued fraction, and let $\left(C_{n \ell}\right)_{n=1}^{\infty}$ be the subsequence of period-ending convergents. The Aitken extrapolants using any step length $p$ in this subsequence,

satisfy

$$
C_{n \ell}^{p \ell *}:=\frac{C_{(n+p) \ell} C_{(n-p) \ell}-C_{n \ell}^{2}}{C_{(n+p) \ell}-2 C_{n \ell}+C_{(n-p) \ell}}, \quad p<n,
$$

$$
C_{n \ell}^{p \ell *}=C_{2 n \ell} .
$$

Proof. Because the continued fraction for $\gamma$ is purely periodic, we have

$$
C_{(n+1) \ell}=\left[b_{1}, \ldots, b_{\ell}, C_{n \ell}\right] .
$$

Thus, by (21) and (22),

$$
C_{(n+1) \ell}=\frac{A_{\ell} C_{n \ell}+A_{\ell-1}}{B_{\ell} C_{n \ell}+B_{\ell-1}},
$$

so that the subsequence is generated by iterating a fractional linear transformation (11), with coefficients $A_{\ell}, A_{\ell-1}, B_{\ell}, B_{\ell-1}$. The initial condition is $C_{\ell}=A_{\ell} / B_{\ell}$ as required, and the coefficients satisfy $B_{\ell} \neq 0$ and $A_{\ell} B_{\ell-1}-A_{\ell-1} B_{\ell}=\operatorname{det} M_{\ell}=$ $(-1)^{\ell} \neq 0$. Therefore the conditions of Theorem 2 are satisfied, and the proof is complete.

As before, repeated extrapolation always doubles the index.

Corollary 2. For every $p \geq 0$, applying Aitken extrapolation to the subsequence $\left(C_{2^{p} n \ell}\right)_{n=1}^{\infty}$ of convergents of the purely periodic regular continued fraction produces the subsequence $\left(C_{2^{p+1} n \ell}\right)_{n=1}^{\infty}$.

By Lemma 1 Aitken extrapolation commutes with adding a constant to the sequence, so a slightly more general result is true.

Corollary 3. The conclusions of Theorem 3 and its corollary hold also if $\gamma>0$ has the form $\gamma=a+\beta$ with an integer $a$ and a reduced quadratic irrational number $\beta$.

3.3. Extrapolation of arbitrary convergents. We now consider Aitken extrapolation in the sequence of convergents of an arbitrary regular continued fraction. As we have seen, extrapolation of convergents does not always produce convergents. We begin by deriving some expressions for the extrapolated convergents, making it possible to state a simple necessary and sufficient condition for an extrapolated convergent to be a convergent.

Theorem 4. Let $\left[b_{1}, b_{2}, \ldots\right]$ be a regular continued fraction, and let $\left(C_{n}\right)_{n=1}^{\infty}$ be its sequence of convergents. For each $n \geq 2$ the Aitken extrapolant $C_{n}^{*}$ is equal to the quotient

$$
C_{n}^{*}=\frac{A_{n+1} B_{n}+A_{n} B_{n-1}}{B_{n+1} B_{n}+B_{n} B_{n-1}},
$$

and to the finite continued fraction of length $2 n$

$$
C_{n}^{*}=\left[b_{1}, b_{2}, \ldots, b_{n}, b_{n+1}, b_{n}, \ldots, b_{2}\right],
$$

and is expressed in homogeneous coordinates by the vector

$$
e_{2}^{T} M_{n}^{T} M_{n+1} .
$$


Therefore $C_{n}^{*}$ belongs to the sequence of convergents if and only if one, and thus all, of (25), (26) or (27) so belongs.

Proof. By (24) of Lemma 4, the estimated contraction rates for the sequence of convergents of a regular continued fraction are

$$
r_{n}=\frac{C_{n+1}-C_{n}}{C_{n}-C_{n-1}}=-\frac{B_{n-1}}{B_{n+1}} .
$$

Thus the extrapolated convergents are

$$
\begin{aligned}
C_{n}^{*} & =C_{n}+\frac{1}{1-r_{n}}\left(C_{n+1}-C_{n}\right) \\
& =\frac{A_{n}}{B_{n}}+\frac{B_{n+1}}{B_{n+1}+B_{n-1}} \cdot\left(\frac{A_{n+1}}{B_{n+1}}-\frac{A_{n}}{B_{n}}\right),
\end{aligned}
$$

and (25) follows upon simplification. Next, write (25) as a quotient of inner products,

$$
\frac{A_{n+1} B_{n}+A_{n} B_{n-1}}{B_{n+1} B_{n}+B_{n} B_{n-1}}=\frac{\left[\begin{array}{ll}
B_{n} & B_{n-1}
\end{array}\right] \cdot\left[\begin{array}{c}
A_{n+1} \\
A_{n}
\end{array}\right]}{\left[\begin{array}{ll}
B_{n} & B_{n-1}
\end{array}\right] \cdot\left[\begin{array}{c}
B_{n+1} \\
B_{n}
\end{array}\right]}=\frac{\left(M_{n} e_{2}\right)^{T} M_{n+1} e_{1}}{\left(M_{n} e_{2}\right)^{T} M_{n+1} e_{2}},
$$

proving (27). Finally, reading from right to left in the matrix product in (27), $M_{n}^{T} M_{n+1}=\left(V_{1} \cdots V_{n}\right)\left(V_{n+1} V_{n} \cdots V_{1}\right)$, we see using homogeneous coordinates that $C_{n}^{*}$ is identical to the $2 n$th convergent of the finite continued fraction of length $2 n+1$,

and this proves (26).

$$
\left[b_{1}, \ldots, b_{n}, b_{n+1}, b_{n}, \ldots, b_{1}\right]
$$

To formulate general sufficient conditions for $C_{n}^{*}$ to be equal to $C_{2 n}$ we identify in the next theorem a particular class of periodic regular continued fractions.

Theorem 5. Let $\gamma$ be a reduced quadratic irrational number, and $\ell$ the length of the primitive period of its regular continued fraction. Assume that $\gamma+\gamma^{\prime}$ is an integer $b_{1}$. Then the partial quotients in the continued fraction for $\gamma$ have the palindrome property

$$
b_{i}=b_{\ell+2-i}, \quad i=2, \ldots, \ell .
$$

Conversely, if the primitive period in the regular continued fraction for the reduced quadratic irrational number $\gamma$ has the palindrome property, then $\gamma+\gamma^{\prime}$ is an integer.

Proof. Let $\gamma$ be a reduced quadratic irrational. If $\gamma+\gamma^{\prime}=b_{1}$, an integer, then since $-1<\gamma^{\prime}<0$ we have $b_{1}=\lfloor\gamma\rfloor$. Hence the regular continued fraction for $\gamma$ has the form $\left[\overline{b_{1}, \ldots, b_{\ell}}\right]$, and the continued fraction algorithm (23) gives $\gamma=b_{1}+1 / \gamma_{1}$ with $\gamma_{1}$ also reduced and

$$
\gamma_{1}=\left[\overline{b_{2}, \ldots, b_{\ell}, b_{1}}\right]
$$

By Galois' theorem [Pe 1929 $\S 23]$, the continued fraction for $-1 / \gamma_{1}^{\prime}$ is obtained by reversing the period of $\gamma_{1}$, that is,

$$
\frac{-1}{\gamma_{1}^{\prime}}=\left[\overline{b_{1}, b_{\ell}, \ldots, b_{2}}\right] \text {. }
$$


But $-1 / \gamma_{1}^{\prime}=b_{1}-\gamma^{\prime}=\gamma$; thus by the uniqueness of the regular continued fraction development, (30) matches the continued fraction for $\gamma$, proving the theorem.

Conversely, if the palindrome property (28) holds, we apply the continued fraction algorithm as before to find $\gamma=b_{1}+1 / \gamma_{1}$, and the continued fracton for $\gamma_{1}=1 /\left(\gamma-b_{1}\right)$ is given by (29). Thus by Galois' theorem

$$
b_{1}-\gamma^{\prime}=\frac{-1}{\gamma_{1}^{\prime}}=\left[\overline{b_{1}, b_{\ell}, \ldots, b_{2}}\right] .
$$

By (28) this is $\gamma$, so that $\gamma+\gamma^{\prime}=b_{1}$, an integer, and the proof is complete.

In fact, if $b_{1}$ is even, it is well known [Pe 1929, §24] that there exists a rational number $d>1$, not the square of a rational number, such that $\gamma=\sqrt{d}+\lfloor\sqrt{d}\rfloor$. When $b_{1}$ is odd, it may be shown that there is a rational number $c$ with $0<c<b_{1}+1$ such that $\gamma$ is the positive root of a quadratic $x^{2}-b_{1} x-c$.

Now we can state sufficient conditions for the Aitken extrapolant of a convergent to be another convergent.

Theorem 6. Let $\gamma$ be a quadratic irrational number, $\left(C_{n}\right)_{n=1}^{\infty}$ the sequence of convergents of its regular continued fraction, and $\ell$ the length of the primitive period. Assume:

(1) The number $\gamma>0$ has the form $\gamma=a+\beta$ with an integer $a$ and a reduced quadratic irrational number $\beta$ such that $\beta+\beta^{\prime}$ is an integer.

(2) For $\ell$ odd, $\ell \mid n$; for $\ell$ even, $(\ell / 2) \mid n$.

If $C_{n}^{*}$ is defined by $C_{n}^{*}=\left(C_{n+1} C_{n-1}-C_{n}^{2}\right) /\left(C_{n+1}-2 C_{n}+C_{n-1}\right)$, then $C_{n}^{*}=C_{2 n}$.

In particular, if $\ell=1$ or $\ell=2$, Aitken extrapolation of the sequence of convergents yields the subsequence of even convergents.

The case of period length $\ell=1$ actually follows from Theorem 1 [MP 1985]: for then all the three-term recursions (21) for the numerators read simply $A_{n}=$ $b_{1} A_{n-1}+A_{n-2}$, every $B_{n}=A_{n-1}$, and the convergents are generated by the iteration $C_{1}=b_{1}, C_{n+1}=b_{1}+1 / C_{n}$.

Proof of Theorem [6. By Lemma 1 and Theorem 5 we may assume that $\gamma$ is a reduced quadratic irrational number and that the primitive period of its continued fraction has the palindrome property.

By Theorem 4, $C_{n}^{*}$ is expressed in homogeneous coordinates by the vector (27).

Assume first that $n=m \ell$, without regard to the parity of $\ell$. Then

$$
M_{n}=M_{\ell}^{m}, \quad M_{n+1}=V_{1} M_{\ell}^{m} .
$$

The matrix $M_{\ell}$ has the factorization $M_{\ell}=H V_{1}$ with $H=V_{\ell} \cdots V_{2}=H^{T}$ by the palindrome property, and Lemma 3 applies to the vector $e_{2}^{T}\left(M_{\ell}^{m}\right)^{T} V_{1} M_{\ell}^{m}$ representing $C_{n}^{*}$. We conclude that this vector is $e_{1}^{T} M_{\ell}^{2 m}=e_{1}^{T} M_{2 m \ell}$. This completes the proof in the case that $\ell \mid n$.

Assume now that $\ell$ is even and $n=(2 m+1) \ell / 2$. The palindrome property now implies that the period matrix $M_{\ell}$ has the factorization

$$
M_{\ell}=K^{T} V_{s} K V_{1}, \quad K=V_{\ell / 2} \cdots V_{2}, \quad s=\ell / 2+1 .
$$


Using again the homogeneous coordinates (27) for $C_{n}^{*}$, we calculate

$$
\begin{aligned}
e_{2}^{T} M_{n}^{T} M_{n+1} & =e_{2}^{T} M_{m \ell+\ell / 2}^{T} M_{m \ell+\ell / 2+1} \\
& =e_{2}^{T}\left(K V_{1}\left(K^{T} V_{s} K V_{1}\right)^{m}\right)^{T} \cdot V_{s} K V_{1} M_{\ell}^{m} \\
& =e_{2}^{T}\left(V_{1} K^{T} V_{s} K\right)^{m} V_{1} K^{T} V_{s} K V_{1} M_{\ell}^{m} \\
& =\left(e_{2}^{T} V_{1}\right) M_{\ell}^{m} M_{\ell} M_{\ell}^{m}=e_{1}^{T} M_{\ell}^{2 m+1}=e_{1}^{T} M_{2 n},
\end{aligned}
$$

completing the proof also in the case that $\ell$ is even and $\ell / 2$ divides $n$.

The difference between Theorem 3 and Theorem [6]may be illustrated as follows. Consider the reduced quadratic irrational number with its purely periodic continued fraction

$$
\xi=\frac{3}{7}+\frac{1}{7} \sqrt{37}=[\overline{1,3,2}] .
$$

According to Theorem 3 extrapolation in the subsequence $\left(C_{3}, C_{6}, C_{9}, C_{12}, \ldots\right)$ produces

$$
C_{6}^{*}=\left(C_{9} C_{3}-C_{6}^{2}\right) /\left(C_{9}-2 C_{6}+C_{3}\right)=C_{12} .
$$

However, the full sequence of convergents of the continued fraction for $\xi$ does not satisfy the hypotheses of Theorem 6 (the period does not have the palindrome property), and its conclusion need not hold. Indeed, by (26) of Theorem 4 we can calculate

$$
C_{6}^{*}=\left(C_{7} C_{5}-C_{6}^{2}\right) /\left(C_{7}-2 C_{6}+C_{5}\right)=[1,3,2,1,3,2,1,2,3,1,2,3],
$$

and this is not equal to $C_{12}$ by the uniqueness of the finite continued fraction.

The factorization $M=H V$ derived in Lemma 3 and used to prove Theorem2 was suggested by the factorization $M_{\ell}=\left(V_{\ell} \cdots V_{2}\right) V_{1}$ used in the proof of Theorem 6 , where $H=V_{\ell} \cdots V_{2}$ is symmetric by the palindrome property. When, as in (31), the sum $\xi+\xi^{\prime}$ is not an integer, the factorization of $M_{\ell}$ provided by Lemma 3 is not related to the factorization $M_{\ell}=V_{\ell} \cdots V_{1}$.

Finally, Theorem 6 by no means describes all cases in which extrapolation of a convergent produces a convergent. Consider

$$
\eta=2+\frac{7}{12} \sqrt{22}=[4,1,2,1,3,1,2,1] .
$$

Then $\ell=8$, and Theorem 6 tells us $C_{4}^{*}=C_{8}, C_{8}^{*}=C_{16}$, and so on. However, using (26) of Theorem 4 again, we also find that

$$
\begin{aligned}
C_{2}^{*} & =[4,1,2,1]=C_{4}, \\
C_{3}^{*} & =[4,1,2,1,2,1]=[4,1,2,1,3]=C_{5},
\end{aligned}
$$

the last line being valid by the qualifying clause [Pe 1929, §9 Satz 2] in the theorem on uniqueness of finite continued fractions.

Examples like these should be regarded as accidents, however, for they do not occur when $n>\ell$.

Theorem 7. Let $\ell \geq 3$ and let $\left(b_{1}, \cdots, b_{\ell}\right)$ be the primitive period of a purely periodic continued fraction having the palindrome property.

Let $n>\ell$ and assume that $n$ is not divisible by $\ell$, nor, if $\ell$ is even, by $\ell / 2$. Then the finite continued fraction

$$
C_{n}^{*}=\left[b_{1}, \cdots, b_{n}, b_{n+1}, \cdots, b_{2}\right]
$$


derived by Aitken extrapolation of $C_{n}$, is not a convergent of $\left[\overline{b_{1}, \cdots, b_{\ell}}\right]$.

Proof. In order for $C_{n}^{*}$ to be equal to some convergent, say $C_{k}$, it is necessary that $C_{k}$ can be written as a continued fraction of length $2 n$. According to the uniqueness theorem [Pe 1929, $\S 9$, Satz 2] there are only three possibilities:

$$
\begin{aligned}
C_{n}^{*} & =C_{2 n}=\left[b_{1}, \cdots, b_{2 n}\right], \\
C_{n}^{*} & =C_{2 n-1}=\left[b_{1}, \cdots, b_{2 n-1}-1,1\right], \quad \text { if } b_{2 n-1}>1, \\
C_{n}^{*} & =C_{2 n+1}=\left[b_{1}, \cdots, b_{2 n}+1\right], \quad \text { if } b_{2 n+1}=1 .
\end{aligned}
$$

We show that none of these cases can occur under the stated hypotheses.

By division $n=q \ell+s$ with $0<s<\ell$, and by hypothesis $q \geq 1$ and $2 s \neq \ell$. Thus $C_{n}^{*}$ has the form

$$
C_{n}^{*}=\left[\cdots, b_{1}, \cdots, b_{s}, b_{s+1}, \cdots, b_{2}, b_{1}, b_{\ell}, \cdots, b_{2}, \cdots\right],
$$

in which the first ellipsis stands for $q$ repetitions of $\left(b_{1}, \cdots, b_{\ell}\right)$ and the last stands for $q-1$ repetitions of $\left(b_{1}, b_{\ell}, \cdots, b_{2}\right)$. By the palindrome property we may rewrite $C_{n}^{*}$ as

$$
C_{n}^{*}=\left[\cdots, b_{1}, \cdots, b_{s}, b_{s+1}, \cdots, b_{2}, b_{1}, b_{2}, \cdots, b_{\ell}, \cdots\right] .
$$

Here the first and last ellipses denote respectively $q$ and $q-1$ repetitions of the primitive period.

We show first that $C_{n}^{*} \neq C_{2 n}$, treating separately the cases $2 s<\ell$ and $2 s>\ell$.

For the case $2 s<\ell$ we display $C_{n}^{*}$ in parallel with $C_{2 n}$, freely using the periodicity of the $b_{i}$ to shift subscripts by multiples of $\ell$ in $C_{2 n}$ when convenient:

$$
\begin{aligned}
& C_{n}^{*}=\left[\begin{array}{lllllllllll}
\cdots & b_{1} & \cdots & b_{s} & b_{s+1} & \cdots & b_{2} & b_{1} & \cdots & b_{\ell} & \cdots
\end{array}\right], \\
& C_{2 n}=\left[\begin{array}{lllllllllll}
\cdots & b_{1} & \cdots & b_{s} & b_{s+1} & \cdots & b_{2 s} & b_{2 s+1} & \cdots & b_{2 s+\ell} & \cdots
\end{array}\right] .
\end{aligned}
$$

Thus, if it were true that $C_{n}^{*}=C_{2 n}$, it would follow that $b_{i}=b_{2 s+i}$ for $1 \leq i \leq \ell$, and hence, by periodicity, for all $i$, contradicting the minimality of the period length $\ell$.

In the case $2 s>\ell$ we have similarly

$$
\begin{aligned}
& C_{n}^{*}=\left[\begin{array}{lllllllllll}
\cdots & b_{1} & \cdots & b_{s} & b_{s+1} & \cdots & b_{2} & b_{1} & \cdots & b_{\ell} & \cdots
\end{array}\right], \\
& C_{2 n}=\left[\begin{array}{lllllllllll}
\cdots & b_{1} & \cdots & b_{s} & b_{s+1} & \cdots & b_{2 s-\ell} & b_{2 s-\ell+1} & \cdots & b_{2 s} & \cdots
\end{array}\right],
\end{aligned}
$$

so that $C_{n}^{*}=C_{2 n}$ implies $b_{i}=b_{2 s-\ell+i}$ for $1 \leq i \leq \ell$, again contradicting the minimality of $\ell$.

This argument shows additionally that $C_{n}^{*} \neq C_{2 n \pm 1}$ when $n>2 \ell$ satisfies our hypotheses, for the proof does not depend on the last $\ell$ partial quotients. It remains only to prove $C_{n}^{*} \neq C_{2 n \pm 1}$ in the case $n=\ell+s$ with $0<s<\ell$ and $2 s \neq \ell$.

If $b_{2 s-1}>1$, we write $C_{2 \ell+2 s-1}$ as a continued fraction of length $2 \ell+2 s$ parallel with $C_{\ell+s}^{*}$. In each display, the initial ellipsis denotes the initial sequence $\left(b_{1}, \cdots, b_{s}\right)$.

$$
\begin{aligned}
& C_{\ell+s}^{*}=\left[\begin{array}{lllllllll}
\cdots & b_{s+1} & \cdots & b_{3} & b_{2} & b_{1} & \cdots & b_{\ell-1} & b_{\ell}
\end{array}\right], \\
& C_{2 \ell+2 s-1}=\left[\begin{array}{lllllllll}
\cdots & b_{s+1} & \cdots & b_{2 s-1} & b_{2 s} & b_{2 s+1} & \cdots & b_{2 s-1}-1 & 1
\end{array}\right] .
\end{aligned}
$$

If these were equal we would have $b_{2 s-1}=b_{3}=b_{\ell-1}=b_{2 s-1}-1$. (In the case $s=1$ the display would be slightly different, and we would deduce $b_{1}=b_{3}=b_{\ell-1}=$ $b_{1}-1$.) 
Finally, if $b_{2 s+1}=1$, then $C_{2 \ell+2 s+1}$ can be written as a continued fraction of length $2 \ell+2 s$. As before, we show only the tail following the initial segment $\left(b_{1}, \cdots, b_{s+1}\right)$ :

$$
\begin{aligned}
C_{\ell+s}^{*} & =\left[\begin{array}{llllclc}
\cdots & b_{s+1} & \cdots & b_{2} & b_{1} & \cdots & b_{\ell}
\end{array}\right], \\
C_{2 \ell+2 s+1} & =\left[\begin{array}{lllllll}
\cdots & b_{s+1} & \cdots & b_{2 s} & b_{2 s+1} & \cdots & b_{2 s}+1
\end{array}\right] .
\end{aligned}
$$

From the equality of these two would follow $b_{2 s}=b_{2}=b_{\ell}=b_{2 s}+1$.

The proof is complete.

3.4. Extrapolation with arbitrary step length. Our final theorem shows that the conclusion of Theorem [ 6 holds for arbitrary step length $k$ not necessarily a multiple of $\ell$.

Theorem 8. Let $\gamma, \ell$ and $n$ satisfy the assumptions of Theorem [6] Then for any $k<n$ the extrapolant of $C_{n}$ with step length $k$,

$$
C_{n}^{k *}=\frac{C_{n+k} C_{n-k}-C_{n}^{2}}{C_{n+k}-2 C_{n}+C_{n-k}},
$$

satisfies $C_{n}^{k *}=C_{2 n}$.

Proof. The proof is in two steps.

In the first step we find a matrix $F$ (depending on $n$ and $k$ ) such that the following expressions for the differences are valid:

$$
C_{n+k}-C_{n}=\frac{-(-1)^{n} F_{12}}{B_{n+k} B_{n}}, \quad C_{n}-C_{n-k}=\frac{-(-1)^{n-k} F_{12}}{B_{n} B_{n-k}} .
$$

These two equations together yield the simple expression $r_{n}^{(k)}=(-1)^{k} B_{n-k} / B_{n+k}$ for the rate estimate; hence the extrapolant is

$$
C_{n}^{k *}=\frac{A_{n+k} B_{n}-(-1)^{k} A_{n} B_{n-k}}{B_{n+k} B_{n}-(-1)^{k} B_{n} B_{n-k}} .
$$

In the second step of the proof, we simplify (34) to obtain (25). The conclusion then follows as in the proof of Theorem 6

We begin with the proof of (33). Define matrices $U_{i}, i=1, \ldots, \ell$ by

$$
U_{i}=\left\{\begin{array}{cl}
V_{i} & \text { if } \ell \mid n, \\
V_{i+\ell / 2} & \text { if } \ell \text { even, } \ell \times n, \quad \ell / 2 \mid n,
\end{array}\right.
$$

and observe that because in the second case $\ell$ is even, the $U_{i}$ have the same palindrome property as the $V_{i}$, viz.

$$
U_{i}=U_{\ell+2-i}, \quad i=2, \ldots, \ell .
$$

Now calculate the forward difference as usual:

$$
C_{n+k}-C_{n}=\frac{A_{n+k} B_{n}-A_{n} B_{n+k}}{B_{n+k} B_{n}} .
$$

The numerator is the determinant of

$$
\left[\begin{array}{rr}
A_{n+k} & B_{n+k} \\
A_{n} & B_{n}
\end{array}\right]=\left[\begin{array}{c}
e_{1}^{T} M_{n+k} \\
e_{1}^{T} M_{n}
\end{array}\right]=\left[\begin{array}{c}
e_{1}^{T} V_{n+k} \cdots V_{n+1} \\
e_{1}^{T}
\end{array}\right] \cdot M_{n} .
$$

If we set

$$
F=V_{n+k} \cdots V_{n+1}
$$


then the determinant (38) is $-(-1)^{n} F_{12}$. This gives the left-hand equation (33). By a similar calculation we find that

$$
C_{n}-C_{n-k}=\frac{-\left(V_{n} \cdots V_{n+1-k}\right)_{12}(-1)^{n-k}}{B_{n} B_{n-k}},
$$

and it remains to show only that the matrix element in the numerator is $F_{12}$. To this end write $k=q \ell+s$ by division; because of Theorem 3 we may assume $0<s<\ell$. Because $n$ is either a multiple of $\ell$ or (when $\ell$ is even) a multiple of $\ell / 2$, we have by the periodicity and the definition (35) of the matrices $U_{i}$ that

$$
F=U_{s} \cdots U_{1}\left(U_{\ell} \cdots U_{1}\right)^{q} .
$$

Now express the matrix element in the numerator of the right side of (40) in terms of the $U_{i}$ : it is

$$
\begin{aligned}
\left(V_{n} \cdots V_{n+1-k}\right)_{12} & =\left(V_{n+1-k} \cdots V_{n}\right)_{21} \\
& =e_{2}^{T} U_{\ell+1-s} \cdots U_{\ell}\left(U_{1} \cdots U_{\ell}\right)^{q} e_{1} \\
& =\left(e_{2}^{T} U_{\ell+1-s}\right) U_{\ell+2-s} \cdots U_{\ell} U_{1}\left(U_{2} \cdots U_{\ell} U_{1}\right)^{q}\left(U_{1}^{-1} e_{1}\right) \\
& =e_{1}^{T} U_{s} \cdots U_{1}\left(U_{\ell} \cdots U_{1}\right)^{q} e_{2}=F_{12} .
\end{aligned}
$$

(The ascending product $U_{\ell+2-s} \cdots U_{\ell}$ in the third line is empty if $s=1$.) The last line follows from the palindrome property (36).

It remains to simplify the expression (34) for the extrapolated quantity. We may assume $k \geq 2$.

By looking at the column vectors in $M_{n+k}=V_{n+k} \cdots V_{n+2} M_{n+1}$ we have, setting $E=V_{n+k} \cdots V_{n+2}$,

$$
\left[\begin{array}{r}
A_{n+k} \\
A_{n+k-1}
\end{array}\right]=E\left[\begin{array}{r}
A_{n+1} \\
A_{n}
\end{array}\right], \quad\left[\begin{array}{r}
B_{n+k} \\
B_{n+k-1}
\end{array}\right]=E\left[\begin{array}{r}
B_{n+1} \\
B_{n}
\end{array}\right] .
$$

Now writing $k=q \ell+s$ with $0<s<\ell$ as before, use periodicity to express

$$
E= \begin{cases}U_{s} \cdots U_{2}, & q=0, s \geq 2, \\ U_{s} \cdots U_{1}\left(U_{\ell} \cdots U_{1}\right)^{q-1} U_{\ell} \cdots U_{2}, & q \geq 1 .\end{cases}
$$

Similarly,

$$
M_{n}=V_{n} \cdots V_{n-k+2} M_{n-k+1},
$$

and this product of $k-1$ matrices $V_{i}$ is simply

$$
V_{n} \cdots V_{n-k+2}=\left\{\begin{array}{ll}
\left(U_{\ell} \cdots U_{1}\right)^{q} U_{\ell} \cdots U_{\ell+2-s}, & s \geq 2, \\
\left(U_{\ell} \cdots U_{1}\right)^{q}, & s=1,
\end{array}\right\}=E^{T} .
$$

by the palindrome property. Therefore the second column of (42) implies

$$
\left[\begin{array}{c}
B_{n-k+1} \\
B_{n-k}
\end{array}\right]=E^{-T}\left[\begin{array}{c}
B_{n} \\
B_{n-1}
\end{array}\right] .
$$

By (41) and (43) we have

$$
\begin{aligned}
& A_{n+k}=E_{11} A_{n+1}+E_{12} A_{n}, \\
& B_{n+k}=E_{11} B_{n+1}+E_{12} B_{n}, \\
& B_{n-k}=E_{21}^{-T} B_{n}+E_{22}^{-T} B_{n-1}
\end{aligned}
$$


We substitute these expressions into (34) and find that

$$
C_{n}^{k *}=\frac{\left(E_{12}+(-1)^{k-1} E_{21}^{-T}\right) A_{n} B_{n}+E_{11} A_{n+1} B_{n}+(-1)^{k-1} E_{22}^{-T} A_{n} B_{n-1}}{\left(E_{12}+(-1)^{k-1} E_{21}^{-T}\right) B_{n}^{2}+E_{11} B_{n+1} B_{n}+(-1)^{k-1} E_{22}^{-T} B_{n} B_{n-1}}
$$

Finally, use the fact that $E_{k}$ is $2 \times 2$ and a product of $k-1$ matrices $V_{j}$, so that $\operatorname{det} E_{k}=(-1)^{k-1}$ and

$$
\begin{aligned}
& \left(E_{k}^{-T}\right)_{22}=\left(E_{k}^{-1}\right)_{22}=(-1)^{k-1}\left(E_{k}\right)_{11}, \\
& \left(E_{k}^{-T}\right)_{21}=\left(E_{k}^{-1}\right)_{12}=-(-1)^{k-1}\left(E_{k}\right)_{12} .
\end{aligned}
$$

Substitute into (44) and simplify to get (25), and the proof is complete.

\section{REFERENCES}

[Br 1991] Claude Brezinski and Michela Redivo Zaglio, Extrapolation Methods: Theory and Practice. New York: Elsevier, 1991. MR 93d:65001

[BL 1986] C. Brezinski and A. Lembarki, "Acceleration of extended Fibonacci sequences." Appl. Numer. Math. 2 (1986) pp. 1-8. MR 87k:65005

[MP 1985] J. H. McCabe and G. M. Phillips, "Aitken Sequences and Generalized Fibonacci Numbers." Math. Comput. 45 (1985) pp. 553-558. MR 87b:41015

[Pe 1929] Oskar Perron, Die Lehre von den Kettenbrüchen. Leipzig: B. G. Teubner, 1929. MR 12:254b MR 16:239e, MR 19:25c (later editions).

[Ph 1984] G. M. Phillips, "Aitken Sequences and Fibonacci Numbers." Amer. Math. Monthly 91 No. 6 (1984), 354-357. MR 85h:65013

Department of Mathematics, 400 Carver Hall, Iowa State University, Ames, Iowa 50011

E-mail address: alex@iastate.edu 